\title{
Endoscopic Submucosal Dissection of a Colonic Calcifying Fibrous Tumor
}

\author{
Jaeyoung Kim, Seongyul Ryu and Yeon-Ji Kim \\ Department of Internal Medicine, St. Vincent's Hospital, College of Medicine, The Catholic University of Korea, Suwon, Korea
}

A 49-year-old woman was referred to our hospital for further treatment due to the suspicion of a submucosal tumor in a routine screening colonoscopy. On colonoscopy, a 1-cm sized subepithelial mass with normal overlying mucosa in the hepatic flexure was found. Endoscopic ultrasonography (EUS) showed a homogenous hypoechoic lesion arising from the second and third layer. We were unable to make a final diagnosis because the lesion showed a small tumor with atypical macroscopic morphology including EUS findings. Therefore, endoscopic submucosal dissection was performed for the diagnostic treatment of the tumor. Submucosal dissection was performed just above the muscle layer, and the tumor was removed completely and reliably without any acute complications such as perforation. Based on histopathological findings, we diagnosed a benign, calcifying fibrous tumor (CFT). The present case is the first report of successful endoscopic diagnosis and treatment of colonic CFT mimicking a submucosal tumor. Clin Endosc 2020;53:487-490

Key Words: Calcifying fibrous tumor; Endoscopic submucosal dissection; Gastrointestinal subepithelial tumors

\section{INTRODUCTION}

A calcifying fibrous tumor (CFT) is a rare, benign soft tissue tumor, characterized by its unique pathologic findings such as the presence of hyalinized collagenous fibrous tissue with psammomatous or dystrophic calcification and focal lymphoplasmocytic infiltrates. ${ }^{1}$ While the location of the tumor may vary, the stomach is the most common (18\%) site, followed by the pleura (9.9\%), while the colon is an extremely rare site $(1.2 \%){ }^{2}$ Most gastrointestinal CFTs are found incidentally during endoscopy or colonoscopy without symptoms. However, using gastroscopy or colonoscopy alone is difficult to

Received: July 16, 2019 Revised: September 11, 2019

Accepted: November 11, 2019

Correspondence: Yeon-Ji Kim

Department of Internal Medicine, St. Vincent's Hospital, College of Medicine, The Catholic University of Korea, 93 Jungbu-daero, Paldal-gu, Suwon 16247, Korea

Tel: +82-31-881-8582, Fax: +82-31-253-8898, E-mail: dr.kimyj@gmail.com ORCID: https://orcid.org/0000-0001-9823-577X

(c) This is an Open Access article distributed under the terms of the Creative Commons Attribution Non-Commercial License (http://creativecommons.org/ licenses/by-nc/3.0) which permits unrestricted non-commercial use, distribution, and reproduction in any medium, provided the original work is properly cited. differentiate it from other submucosal tumors, which have malignant potential. Endoscopic ultrasonography (EUS) also has difficulties in distinguishing between CFT and other premalignant subepithelial tumors. Therefore, complete resection is the standard strategy for accurate diagnosis and treatment. ${ }^{2}$ To date, most resections have been surgical resections. In the case of gastric CFT, resection through endoscopic submucosal dissection (ESD) has been reported in one case. ${ }^{3}$ However, there is no report of endoscopic resection of CFT in the colon. Here, we report a case of successful treatment of subepithelial CFT in the large intestine with ESD.

\section{CASE REPORT}

A 49-year old woman was referred to our hospital for a small colonic subepithelial tumor (submucosal tumor) detected by screening colonoscopy. Mucosal biopsy specimens from other clinics showed no specific findings. She had no significant medical history or abdominal symptoms. The physical examination and laboratory findings were normal.

A yellowish protruding lesion about $1 \mathrm{~cm}$ in diameter was 
observed in the hepatic flexure on colonoscopy. The surface mucosa was normal, and the lesion showed a hard consistency. The pillow and rolling signs were positive. These findings were suggestive of a subepithelial tumor. (Fig. 1). EUS was performed for the differential diagnosis of a submucosal tumor and visualized a 1-cm sized homogenous hypoechoic lesion arising in the second and third layer of colonic wall, with the first layer preserved (Fig. 2). There was post-acoustic shadowing of a slightly hyperechoic foci inside the lesion (red arrow). Due to the small lesion size, it was difficult to perform EUS-guided fine-needle aspiration (EUS-FNA), and premalignant subepithelial tumors such as neuroendocrine tumors could not be excluded. The patient had psychological anxiety regarding the unclear tumorous lesion. We decided to perform ESD for diagnostic treatment. Hypertonic sa-

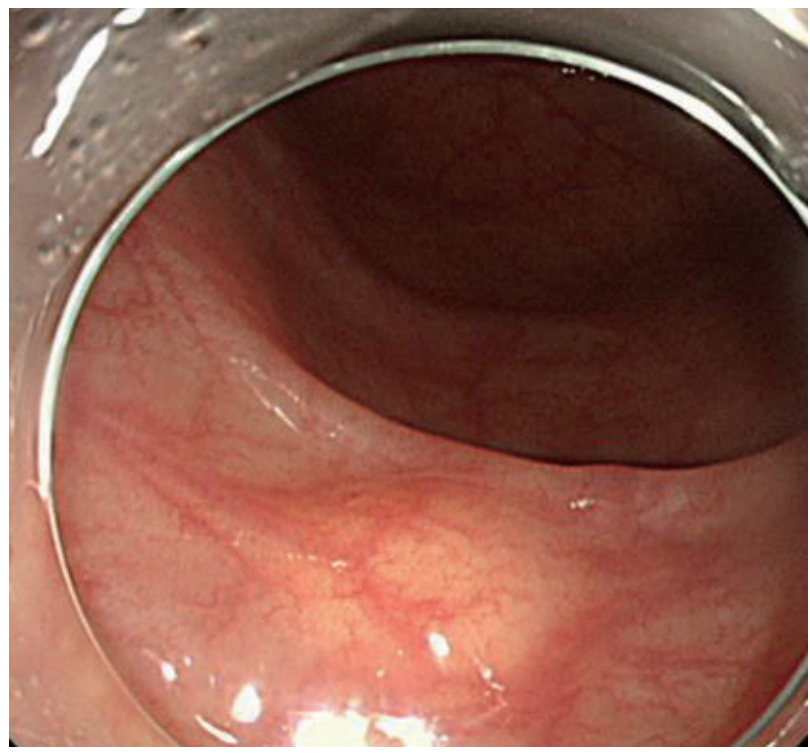

Fig. 1. Endoscopic view of colonic subepithelial tumor. line-epinephrine solution was injected into the submucosa to elevate the lesion. A dual knife (KD-650U; Olympus, Tokyo, Japan) was used for dissecting the submucosa. The lesion was completely resected, and no residual lesion remained (Fig. 3). The tumor was well-demarcated and whitish. Deep mucosal injury occurred during the procedure and an oozing visible vessel was observed. Five hemoclips were used for hemostasis, and bleeding was successfully controlled. There were no specific complications after the procedure, and the patient was discharged the next day.

Histologically, a relatively clear, unencapsulated mass was observed. Hematoxylin and eosin stained specimens showed hypocellular spindle cell proliferation, abundant hyalinized collagen, and prominent lymphoplasmacytic inflammatory

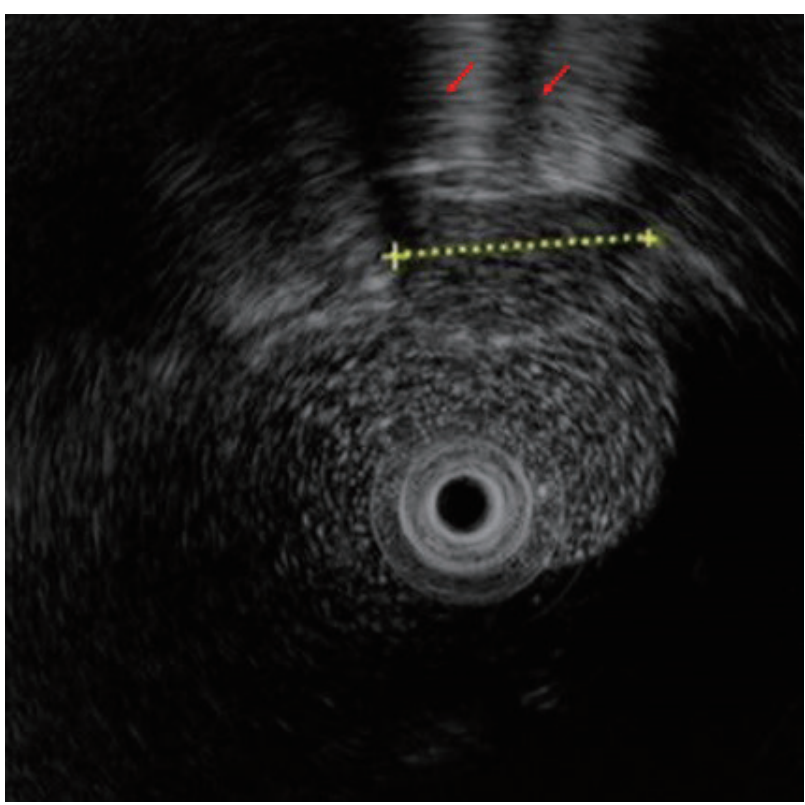

Fig. 2. Endoscopic ultrasonographic evaluation of colonic subepithelial tumor.
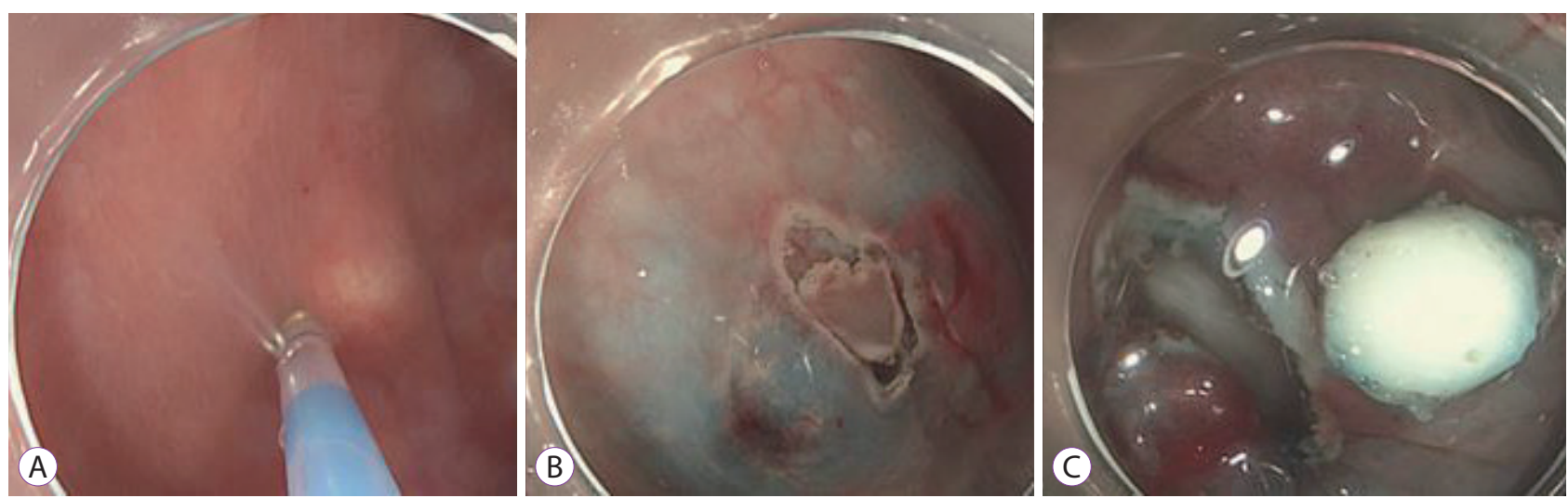

Fig. 3. (A) Injection by conventional needle. (B) Circumferential incision. (C) After completion of the whole procedure. 

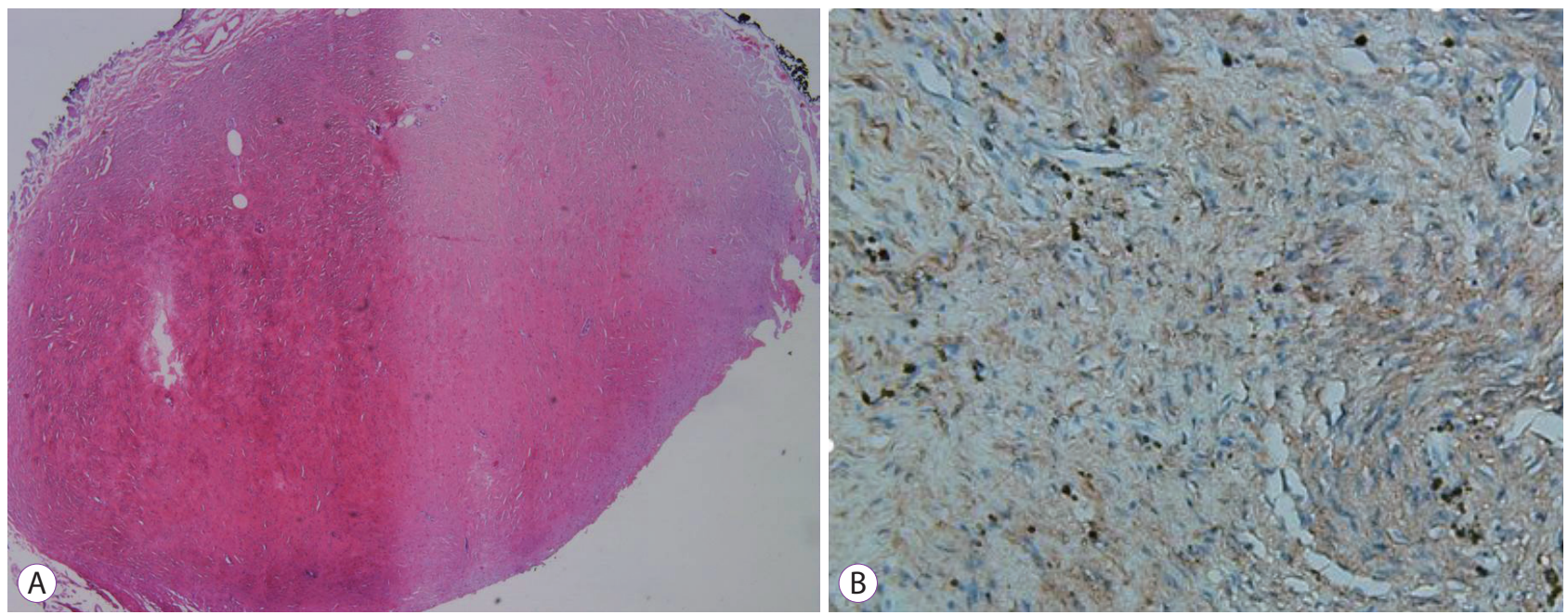

Fig. 4. Pathologic findings of the tumor. (A) Hematoxylin and eosin stained specimens showing hypocellular spindle cell proliferation, abundant hyalinized collagen, and prominent lymphoplasmacytic inflammatory infiltration. (B) Immunohistochemical staining showing tumor cells that were negative for S100.

infiltration. Immunohistochemical staining for Desmin, C-kit, and S100 showed negative results. These histopathologic findings were consistent with CFT (Fig. 4A, B).

\section{DISCUSSION}

CFT is a rare benign fibrous tumor that was first reported by Rosenthal et al. ${ }^{4}$ CFT occurs in various parts of the body. The site of disease can encompass a very large area of the body including the stomach (18\%), small intestine (8.7\%), pleura $(9.9 \%)$, neck $(6.2 \%)$, and mesentery $(5 \%)$ in addition to others, but it is very rarely seen in the large intestine $(1.2 \%)^{2}{ }^{2}$ CFT in the form of a submucosal tumor in the large intestine has been mostly found in the rectum. In the case of intestinal CFT, a submucosal tumor is the most common form, so it is necessary to distinguish it from other unusual tumors (e.g., metastatic tumors or lymphoma) or premalignant submucosal tumors, such as a neuroendocrine tumor. ${ }^{5}$ Therefore, in this case, EUS was performed for discrimination, and a 1-cm sized homogenous hypoechoic lesion that occurred in the second layer and third layer was observed on EUS. However, several previous studies reported the unsatisfactory accuracy of EUS (about $43 \%-79 \%$ ) compared with that of pathologic diagnosis in gastrointestinal submucosal tumors. ${ }^{6,7}$ Although an acoustic shadow with calcification was a rare finding in a neuroendocrine tumor, because of the small mass size, accurate diagnosis was difficult. Indeed, there have been reports of metastasis from neuroendocrine tumors less than $1 \mathrm{~cm}$; furthermore, neuroendocrine tumors from the midgut, including the ascending colon, are known to be more likely to have distant metastasis than rectal neuroendocrine tumors. ${ }^{8}$ We considered performing pathological evaluation, such as EUS-FNA, for confirmation but the lesion size was about $10 \mathrm{~mm}$ and thus, too small to perform EUS-FNA in this case.

Currently, new endoscopic resection techniques such as ESD have been developed and effective endoscopic treatment of subepithelial tumor has become possible. We decided to perform ESD for diagnostic treatment. Through ESD, the lesion was completely resected and histologically confirmed by CFT. Previously, surgical treatment was the standard for submucosal tumors in the gastrointestinal tract. However, in the case of a submucosal tumor, it is more difficult to locate the lesion through endoscopy as in the surgical method, and the complications due to intestinal resection and the risk of surgery itself are often obstacles to the treatment. ${ }^{9}$

All colonic CFTs reported so far were confirmed and treated by open surgical resection. Only one case of gastric CFT successfully treated with ESD has been reported. ${ }^{10}$ To date, there has been no report of endoscopic treatment of colon CFT, including ESD. The present case is the first report of successful endoscopic diagnosis and treatment of colonic CFT mimicking a submucosal tumor. This case is of significance for showing that a colonic CFT can be well treated with endoscopic procedures. If a submucosal tumor of the colon is found in a suspected case of CFT, ESD may be considered an effective treatment.

Conflicts of Interest

The authors have no financial conflicts of interest. 
ORCID

Jaeyoung Kim: https://orcid.org/0000-0003-3458-1571

Seongyul Ryu: https://orcid.org/0000-0001-9099-937X

\section{REFERENCES}

1. Fetsch JF, Montgomery EA, Meis JM. Calcifying fibrous pseudotumor Am J Surg Pathol 1993;17:502-508.

2. Chorti A, Papavramidis TS, Michalopoulos A. Calcifying fibrous tumor: review of 157 patients reported in international literature. Medicine (Baltimore) 2016;95:e3690.

3. Li BJ, Yang XD, Chen WX, Shi YH, Nie ZH, Wu J. Calcifying fibrous tumor of stomach: a case report. Medicine (Baltimore) 2017;96:e8882.

4. Rosenthal NS, Abdul-Karim FW. Childhood fibrous tumor with psammoma bodies. Clinicopathologic features in two cases. Arch Pathol Lab Med 1988;112:798-800

5. Pezhouh MK, Rezaei MK, Shabihkhani M, et al. Clinicopathologic study of calcifying fibrous tumor of the gastrointestinal tract: a case series. Hum Pathol 2017;62:199-205.

6. Kwon JG, Kim EY, Kim YS, et al. [Accuracy of endoscopic ultrasonographic impression compared with pathologic diagnosis in gastrointestinal submucosal tumors]. Korean J Gastroenterol 2005;45:88-96.

7. Hwang JH, Saunders MD, Rulyak SJ, Shaw S, Nietsch H, Kimmey MB. A prospective study comparing endoscopy and EUS in the evaluation of GI subepithelial masses. Gastrointest Endosc 2005;62:202-208.

8. Quaedvlieg PF, Visser O, Lamers CB, Janssen-Heijen ML, Taal BG. Epidemiology and survival in patients with carcinoid disease in The Netherlands. An epidemiological study with 2391 patients. Ann Oncol 2001;12:1295-1300.

9. Ponsaing LG, Kiss K, Loft A, Jensen LI, Hansen MB. Diagnostic procedures for submucosal tumors in the gastrointestinal tract. World J Gastroenterol 2007;13:3301-3310.

10. Ogasawara N, Izawa S, Mizuno M, et al. Gastric calcifying fibrous tumor removed by endoscopic submucosal dissection. World J Gastrointest Endosc 2013;5:457-460. 